\title{
Temperature dependence of the deviations from bulk behavior in ultrathin polymer films
}

\author{
Simone Napolitano, ${ }^{*}$ Veronica Lupaşcu and Michael Wübbenhorst \\ *simone.napolitano@fys.kuleuven.be
}

To extract quantitative information about the relaxation spectra isothermal dielectric spectra were analyzed by the empirical Havriliak-Negami (HN) function

$$
\varepsilon(\omega)=\varepsilon_{\infty}+\frac{\Delta \varepsilon}{\left[1+\left(i \omega \tau_{H N}\right)^{a}\right]^{b}}
$$

where $\varepsilon_{\infty}$ is the value dielectric constant in absence of polarization processes (low temperature and high frequencies limit), $\Delta \varepsilon, \tau_{\mathrm{HN}}, a, b$ indicate respectively the dielectric strength, relaxation time and shape parameters related to the width and the asymmetry of the loss curves. To take into account the contribution due to the conductivity, the term $-i \varepsilon_{0}^{-l} \sigma \omega^{-n}$ (where $\varepsilon_{0}=8.854 \mathrm{pF} / \mathrm{m}$ is the vacuum permittivity and $n \leq 1$ ) was added to Eq. (2.1).

The most probable of the structural relaxation times, $\tau_{\alpha}$, associated to $v_{\max }$, the frequency of the maximum of the dispersion peak $\left(2 \pi \tau_{\alpha} \cdot v_{\max }=1\right)$, is calculated from the relaxation time obtained in $\mathrm{Eq}(\mathrm{S} .1)$ via the shape parameters $a$ and $b$ :

$$
\tau_{\alpha}=\tau_{H N}\left\{\sin \left[\frac{a \pi}{2+2 b}\right]\right\}^{a}\left\{\sin \left[\frac{a b \pi}{2+2 b}\right]\right\}^{-a}
$$

Upon reduction of the thickness, in addition to the features described in the text (increase of $\tau_{\alpha}$, reduction of $\Delta \varepsilon$, i.e. the area below the loss peak) a broadening of the peaks was observed, as already described by others. $^{1-3}$ In terms of the HN function the broadening is related to a reduction of the shape parameter $a$, see Figure S.1.

In figure S.2 and S.3 we plotted the thickness dependence of isothermal spectra at three selected thickness: one thick film $(174 \mathrm{~nm})$ a film in which the values of $\Delta \varepsilon$ and $a$ are reduced compared to the bulk, while no confinement effects are observed for $\tau_{\alpha}()$ and a thinner film $(8 \mathrm{~nm})$ where the shift of the structural peak is clearly shown. To allow a direct comparison, conductivity contributions were subtracted in the spectra at lower temperatures. Note how the separation between the loss peaks reduces at high temperature, as discussed in the text. 
Figure S.1

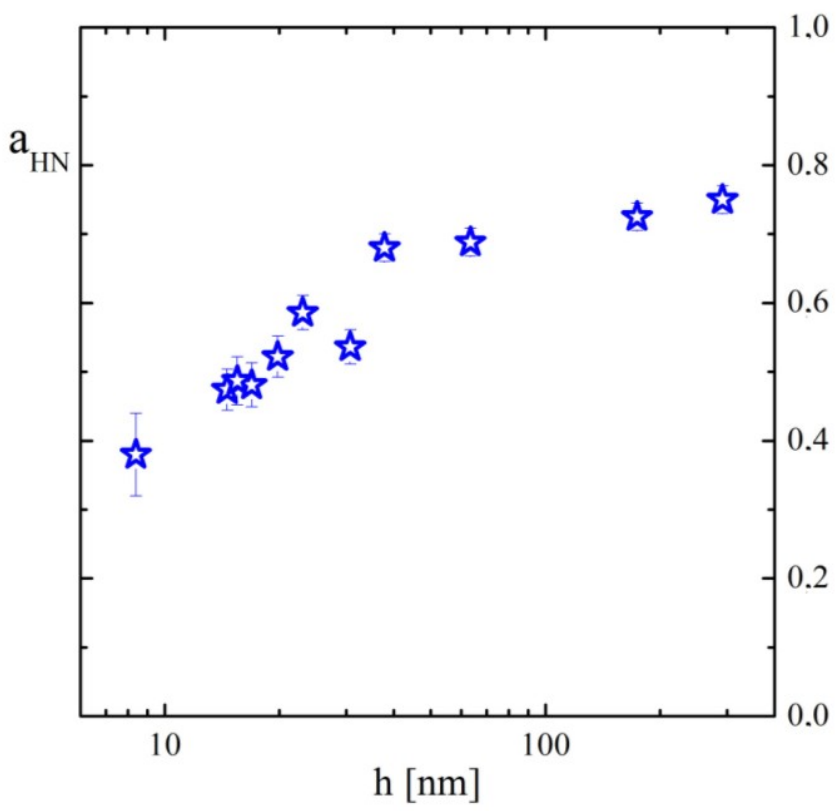

Figure S.2 and S.3
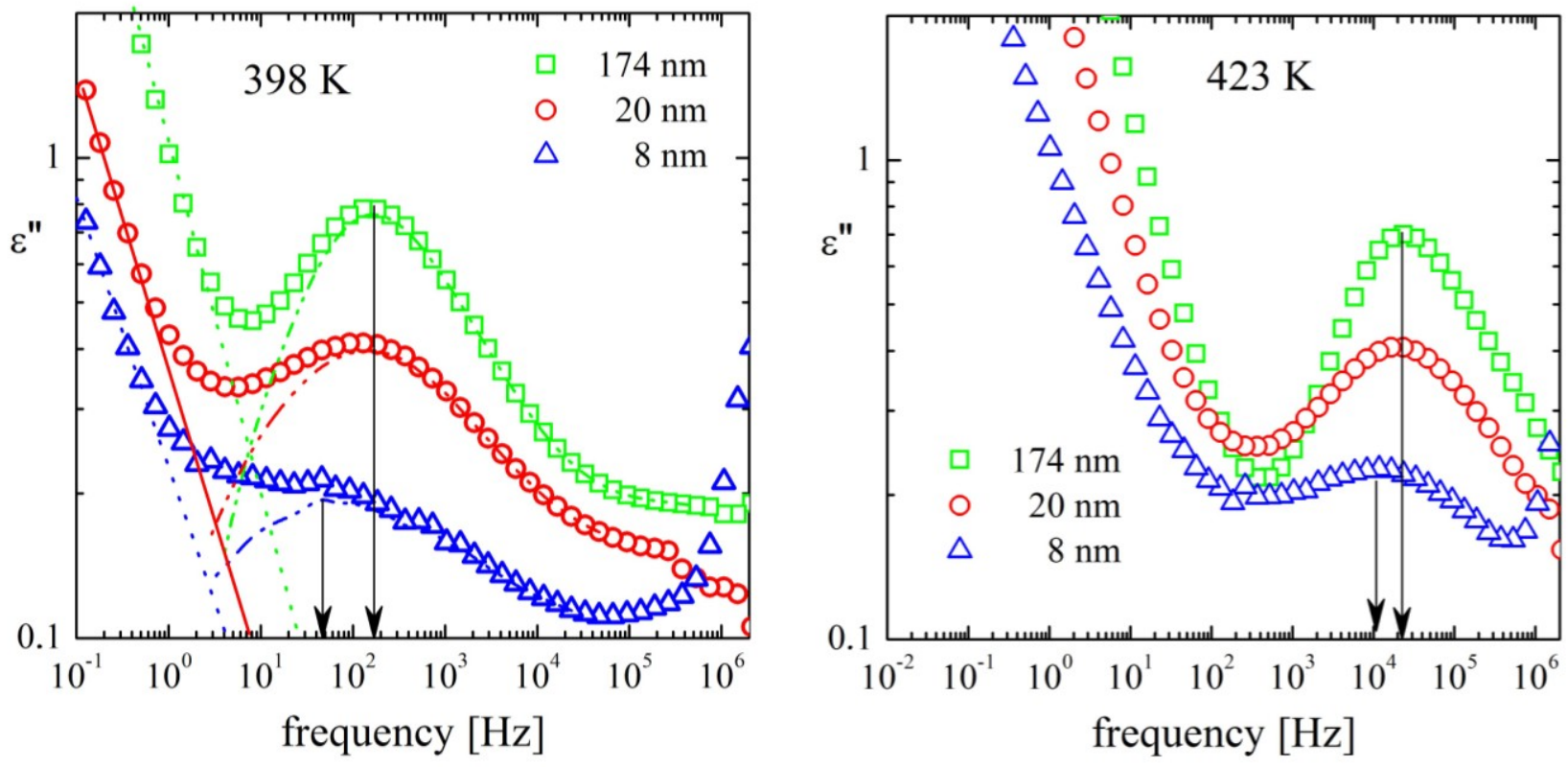

(1) Fukao, K.; Miyamoto, Y. Europhysics Letters 1999, 46, 649.

(2) Fukao, K.; Uno, S.; Miyamoto, Y.; Hoshino, A.; Miyaji, H. Physical Review E 2001, 6405.

(3) Napolitano, S.; Prevosto, D.; Lucchesi, M.; Pingue, P.; D'Acunto, M.; Rolla, P. Langmuir 2007, 23, 2103. 